\section{ORIGINAL RESEARCH}

J.M. Lupo

S. Cha

S.M. Chang

S.J. Nelson

\title{
Analysis of Metabolic Indices in Regions of Abnormal Perfusion in Patients with High-Grade Glioma
}

\begin{abstract}
BACKGROUND AND PURPOSE: MR spectroscopic imaging (MRSI) and dynamic susceptibility-contrast MR imaging (DSC-MR imaging) are functional in vivo techniques for assessing tumor metabolism and vasculature characteristics. Because tumor hypoxia is influenced by tortuous, degraded, swollen, and angiogenic tumor vasculature, regions of abnormal perfusion parameters should coexist with changes in lactate and creatine metabolite levels.
\end{abstract}

\begin{abstract}
MATERIALS AND METHODS: DSC-MR imaging and lactate-edited MRSI were performed on 38 treatment-naive patients with high-grade gliomas (17 grade III, 21 grade IV) before surgical diagnosis. Regions of abnormal perfusion were determined from peak height and percent recovery maps for each voxel within the spectroscopic imaging volume. Choline, creatine, and lactate levels within voxels experiencing only abnormal peak height (aPH), only abnormal recovery (aRec), and both abnormal peak height and recovery (aPH + aRec) were determined and compared to the surrounding T2 hyperintensity (T2h) and normal-appearing white matter.
\end{abstract}

RESULTS: There were decreasing trends in volume from aPH to aRec to aPH + aRec regions for both grade III and grade IV gliomas. Grade IV gliomas exhibited significantly elevated choline in all abnormal perfusion regions, with reduced creatine and increased lactate in the aRec region relative to the surrounding T2h. Grade III gliomas showed trends toward increased creatine within the aPH region and reduced levels within the aRec region.

CONCLUSION: Depressed creatine and elevated lactate levels confirmed the lack of oxygenation within regions of compromised vascular integrity. Identification of regions with leaky or dense vasculature and metabolic markers of hypoxia and cellular proliferation could be useful in determining the more aggressive part of the tumor for targeting, monitoring, and assessing effects of treatment.

W ith the increase in tumor burden during neoplastic transformation, the respiratory capacity decreases as the vascular supply is no longer adequate to support the increasing metabolic demands of the rapidly proliferating tumor cells. Regional hypoxia then ensues, resulting in 2 effects: 1) the increased reliance on glycolysis for energy production, and 2) the upregulation of vasoactive endothelial growth factor and promotion of new blood vessel formation from the existing vasculature, a phenomenon known as angiogenesis. ${ }^{1-4}$ The new vessels that are formed often lack the complex structure of the normal brain vasculature, increasing endothelial permeability. Tumor growth can also damage the existing vasculature by elevating interstitial pressure, causing large transport distances in the interstitium, and promoting blood-brain barrier $(\mathrm{BBB})$ breakdown, which results in microvascular leakage. ${ }^{5,6}$ As the end product of nonoxidative glycolysis, elevated lactate levels may also be markers of reduced cellular oxygenation and hypoxia in these lesions. ${ }^{1,7-9}$ However, the concen-

Received September 18, 2006; accepted after revision January 16, 2007.

From the Departments of Radiology (J.M.L., S.C., S.J.N.) and Neurological Surgery (S.C., S.M.C.) and the Program in Bioengineering (S.J.N.), University of California, San Francisco, Calif.

The research was supported by the following grants: SPORE P50 CA97297, National Institutes of Health/National Cancer Institute, Washington, DC; and LSIT-01-10107, University of California Discovery Grants with GE Healthcare, University of California Office of the President, Berkeley, Calif

Paper previously presented in part at: 13th Annual Meeting of the International Society for Magnetic Resonance in Medicine, May 13-17, 2005; Miami, Fla.

Please address correspondence to Janine Lupo, PhD, UCSF Radiology, Box 2532, Byers Hall, 1700 4th St, San Francisco, CA 94158; e-mail: janinel@mrsc.ucsf.edu

DOI 10.3174/ajnr.A0586 tration of lactate may involve complicated interplay between hemodynamics and cellular proliferation and energetics. In addition, creatine levels have also been associated with energy metabolism and bioenergetics in tumors. ${ }^{10-12}$

MR spectroscopic imaging (MRSI) and dynamic susceptibility-contrast perfusion MR imaging (DSC-MR imaging) are functional in vivo techniques capable of quantitatively assessing metabolic and vascular activity in tumors. Numerous studies have implemented MRSI to extract information about brain tumor cellularity and cell membrane breakdown, cellular energetics, neuronal activity, and macroscopic necrosis through its ability to distinguish signals from choline, creatine, $\mathrm{N}$-acetylaspartate, lactate, and lipid molecules. ${ }^{11-16}$ DSC-MR imaging facilitates analysis of hemodynamic parameters by tracking an intravenous contrast agent bolus through the vasculature, resulting in a $\mathrm{T} 2{ }^{\star}$ relativity curve proportional to concentration and, therefore, vascular density. Various groups have compared relative cerebral blood volume (rCBV) and/or permeability values derived from DSC-MR imaging with metabolic indices in low- and high-grade brain tumors. ${ }^{17-22}$ However, these studies usually analyze only the central contrast-enhancing portion of the tumor or region with maximal rCBV and do not use spectroscopic imaging sequences sensitive to lactate detection. ${ }^{19,23-29}$

Previous studies have shown that voxel-by-voxel comparison of percent recovery and peak-height values obtained from a nonparametric model can be easily used to characterize independently regions of BBB breakdown and increased vessel volume due to angiogenesis. ${ }^{30}$ In addition, regions of abnormal perfusion were found outside the contrast-enhancing vol- 
ume in both grade III and grade IV gliomas. ${ }^{30,31}$ By combining lactate-edited 3D MRSI and perfusion-weighted imaging, this study aims to investigate metabolic levels within distinct regions of 1) abnormal vessel volume with intact vessels and 2) structurally compromised vessels of normal density in both grade III and grade IV gliomas. Because heightened cell density and proliferation require the recruitment of additional vessels for oxygenation, areas of elevated vessel volume are hypothesized to experience increased choline levels. For regions that exhibit insufficient perfusion of oxygen due to $\mathrm{BBB}$ breakdown, reductions in creatine and heightened lactate levels that mark anaerobic respiration are expected, with larger changes projected for grade IV gliomas.

\section{Methods}

\section{Patients}

MR imaging was performed on 38 patients with newly diagnosed brain tumor ( 15 women, 23 men) immediately before undergoing surgical resection at our institution. All patients provided informed written consent using a protocol approved by our institutional review board. Patients had no clinical history of prior treatment. Histopathologic analysis of the resected tissue confirmed the diagnosis of 17 grade III gliomas (mean age, 41 years; median age, 36 years; age range, 19-69 years; 11 anaplastic astrocytomas, 2 oligodendrogliomas, 4 oligoastrocytomas) and 21 grade IV gliomas (mean age, 55 years; median age, 58 years; age range, $27-75$ years; all glioblastoma multiforme) as classified by the World Health Organization II criteria.

\section{MR Imaging and Spectroscopic Acquisition}

MR imaging examinations were performed on a 1.5T Signa EchoSpeed scanner (GE Healthcare Technologies, Waukesha, Wis) using a standard quadrature head coil. The MR imaging protocol consisted of an axial fluid-attenuated inversion recovery (FLAIR, 10,000/148/2200 $\mathrm{ms}[\mathrm{TR} / \mathrm{TE} / \mathrm{TI}])$ or axial $3 \mathrm{D}$ fast spin-echo T2-weighted imaging (FSE; 3000/102 ms [TR/TE]), dynamic susceptibility-weighted gradient-echo echo-planar imaging (EPI; 1000-1250/54 ms [TR/TE]; flip angle, $35^{\circ}$ ), and postcontrast $3 \mathrm{D}$ spoiled gradient-recalled (SPGR, 34/8 ms) T1-weighted imaging. The T2-FLAIR, FSE, and postcontrast T1-SPGR images were acquired and used to define regions of T2 hyperintensity and $\mathrm{T} 1$ enhancement.

For the dynamic EPI series, the location and size of the tumor and the position of the superior and inferior margins were determined from the T2-weighted FLAIR or FSE images. Seven or 8 slices were selected to cover most of the tumor volume. Slice thickness ranged from 3 to $5 \mathrm{~mm}$ and slice gap of 0 to $2 \mathrm{~mm}$. A standard dose of 0.1 $\mathrm{mmol} / \mathrm{kg}$ body weight of gadopentetate dimeglumine (Gd-DTPA) was injected intravenously with an MR imaging-compatible power injector at a rate of $5 \mathrm{~mL} / \mathrm{s}$, followed immediately by a $20-\mathrm{mL}$ continuous saline flush. A series of $60 \mathrm{~T} 2{ }^{\star}$-weighted multislice image sets (with a $26 \times 26 \mathrm{~cm}^{2}$ in-plane FOV and $128 \times 128$ acquisition matrix) were acquired every $1-1.25$ seconds during the first pass and recirculation phase of the contrast agent bolus.

3D J-difference lactate-edited MRSI data were acquired using a Point-Resolved Spectroscopy (PRESS) volume localization (TE/TR, 144/1000 ms) with dual band selective inversion with gradientdephasing (BASING) pulses developed previously in our laboratory. ${ }^{32}$ Because the lactate resonance is confounded by the presence of lipid at $0.9-1.3 \mathrm{ppm}$ in a conventional MR spectroscopy acquisition,
J-difference spectral editing methods were implemented to achieve full sensitivity of the lactate doublet using 2 acquisition cycles per phase-encode step to discriminate between coupled and uncoupled spins. ${ }^{31-33}$ Chemical shift selective saturation and very selective saturation (VSS) pulses were applied for water and outer volume suppression, respectively. The VSS pulses were able to provide a sharper PRESS-selection volume and reduce chemical shift misregistration artifacts. ${ }^{34}$ The excited PRESS volume (usually $200-300 \mathrm{~cm}^{3}$ ) was positioned to cover as much of the lesion as possible while avoiding materials such as bone and subcutaneous fat that would complicate shimming and water suppression. Contralateral normal tissue was also included as a reference for postprocessing of metabolites and region definition. ${ }^{20} \mathrm{~K}$-space sampling was restricted to the central ellipsoidal region within a $12 \times 12 \times 8$ phase-encode matrix to shorten the acquisition time to approximately half that of the full $k$-space sampling. ${ }^{33}$ This technique allowed separation of lactate from lipid within the same total acquisition time ( $\sim 17$ minutes).

\section{Image and Spectra Postprocessing}

All images were transferred off-line to a UNIX workstation (Sun Microsystems, Mountain View, Calif) and processed by using in-house programs created in C and IDL (Research Systems, Boulder, Colo) programming languages or with Matlab 6.5 software (MathWorks, Natick, Mass). The metabolite and perfusion images were rigidly aligned to a postcontrast injection 3D SPGR image and resampled to a $32 \times 32$ grid in-plane with a $16 \times 16 \mathrm{~cm}^{2} \mathrm{FOV}$. When necessary, the perfusion dataset was aligned to the anatomic images using affine and perspective transformations or nonrigid B-spline warping ${ }^{35}$ by maximization of normalized mutual information. ${ }^{36}$ The pixel size of the perfusion data was increased to $5 \times 5 \mathrm{~mm}^{2}$ resolution so that the observed perfusion signal-intensity changes had sufficient signal-tonoise ratio to be analyzed reliably on a voxel-by-voxel basis, and the degree of interpolation of the metabolite maps was minimized.

The resampled $\mathrm{T}^{*}$ signal-intensity time curves acquired during the first pass of the gadolinium bolus were converted to the change in relaxation rate $\left(\Delta \mathrm{R} 2^{*}\right)$. The precontrast baseline signal intensity was established from 6 image volumes acquired before contrast injection. Because the relative Gd-DTPA concentration is proportional to the $\triangle \mathrm{R} 2^{\star}$ curve, a plot of the relative concentration of Gd-DTPA in tissue over time was obtained for each voxel. Peak height and percent recovery of the postbolus signal intensity from the peak were calculated from the $\Delta \mathrm{R} 2^{\star}$ curve of the perfusion data for each voxel within the PRESS localized volume. Peak height values were normalized to the peak of a model curve function derived from normal-appearing brain based on histogram analysis of the precontrast echo-planar images. ${ }^{30}$ Voxels with peak-height values greater than twice the model curve were classified as having abnormal peak height, whereas those whose postbolus concentration recovered less than $75 \%$ from the peak concentration were considered to have abnormal recovery.

The 3 abnormal perfusion regions comprised voxels experiencing 1) abnormal peak height but not abnormal recovery ( $\mathrm{aPH}), 2$ ) abnormal recovery but not abnormal peak height ( $\mathrm{aRec}$ ), or 3) both abnormal peak height and recovery $(\mathrm{aPH}+\mathrm{aRec})$. Regions within the T2 lesion that were within the PRESS localization volume were identified by manual delineation on the FLAIR or FSE images. The subregion that we defined as $\mathrm{T} 2 \mathrm{~h}$ and that we used for comparison of metabolite levels excluded regions of contrast enhancement (defined from regions of interest on the SPGR), abnormal perfusion, and macroscopic necrosis. Normal-appearing white matter (NAWM) regions were determined from visual inspection of spectral voxels and selection of 


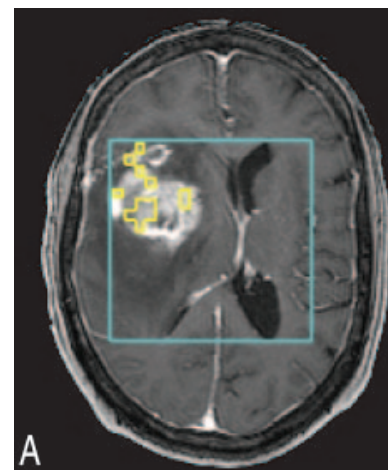

$\mathrm{aPH}$

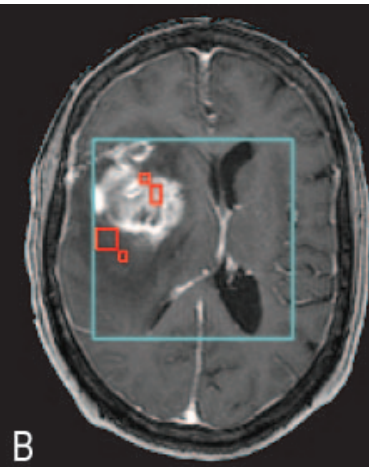

B

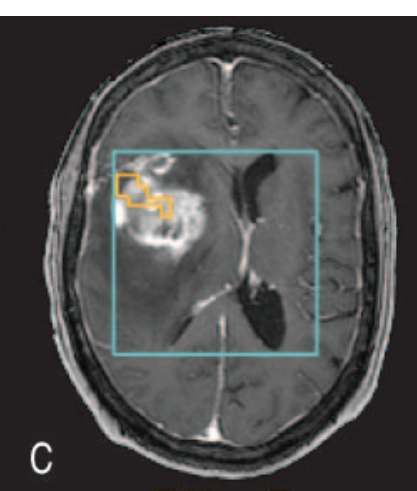

aRec
C

aPH+aRec

Fig 1. Heterogeneity in the distribution of abnormal perfusion regions within a representative grade IV glioma. $A$, The region describing only abnormal peak height (aPH) and normal recovery patterns. $B$, The region consisting of only abnormal recovery (aRec) with normal peak height values. $C$, The intersection of both abnormal peak height and abnormal recovery $(\mathrm{aPH}+\mathrm{aRec})$ is displayed. $D$ and $E$, The T2 hyperintensity lesion (T2h), which excludes all regions of abnormal perfusion, contrast enhancement, and macroscopic necrosis, and contralateral normal appearing white matter (NAWM).

those only located entirely in white matter contralateral to the tumor, without partial voluming with other tissue or contaminated by artifacts. Areas of signal dropout due to susceptibility on the echo-planar images were excluded from all regions. Abnormal perfusion, T2h, and NAWM regions overlaid on postcontrast T1-weighted images are shown in Fig 1 for an example of a patient with grade IV glioma. Volumes of aPH, aRec, aPH $+\mathrm{aRec}$, and $\mathrm{T} 2$ hours were calculated and normalized according to the entire $\mathrm{T} 2$ lesion volume within the perfusion images.

3D MRSI data were quantified off-line using software developed in our laboratory to estimate the levels of choline, creatine, and lactate using the summed and difference spectra from the 2 acquisition cycles. ${ }^{14}$ Baseline, phase, and frequency correction parameters from the summed spectra were applied to the difference spectra. Spectral values were determined from the height of each metabolite peak and normalized relative to the noise levels of the right-hand end of the spectra.

\section{Statistical Analysis}

Metabolite levels were calculated within each region for each patient individually and then averaged across patients in each cohort. Voxels from all patients of the same grade were also combined for each region, and mean values of parameters were computed. Statistical significance for differences in metabolite levels between the various regions of the same patient group were quantified by using a signedrank test, whereas metabolite levels for a given region compared between patient groups were considered significant using a Wilcoxon ranked sum test. $P$ values less than .05 were considered significant. Data are expressed as mean $\pm \mathrm{SD}$.

\section{Results}

All patients had large regions of T2 hyperintensity that were assumed to correspond to the area at risk for increased meta-
Table 1: Volumes of abnormal perfusion and T2h regions expressed as a percentage of the total T2 lesion volume within the PRESS box

\begin{tabular}{lccccc}
\hline & \multicolumn{2}{c}{ Grade III } & & \multicolumn{2}{c}{ Grade IV } \\
\cline { 2 - 3 } \cline { 5 - 6 } & & No. & & No. \\
Region & Volume (\%) & Patients & & Volume (\%) & Patients \\
\hline aPH & $11.0 \pm 10.5$ & 15 & & $16.3 \pm 11.6$ & 20 \\
aRec & $10.9 \pm 17.5$ & 12 & & $12.3 \pm 12.7$ & 20 \\
aPH+aRec & $1.6 \pm 2.3$ & 10 & & $5.1 \pm 6.1$ & 19 \\
T2h & $74.4 \pm 19.0$ & 16 & & $50.4 \pm 17.3$ & 21 \\
\hline
\end{tabular}

Note:- $-\mathrm{aPH}$ indicates the region of abnormal peak height with normal recovery; aRec, the region of abnormal recovery with normal peak height.

bolic and vascular abnormality. Seven of the 17 patients with grade III lesions and all 23 patients with grade IV lesions exhibited enhancement on the T1-weighted postcontrast SPGR images. Although the intention was to cover the entire region of T2 hyperintensity for each lesion, the functional MR and spectroscopic imaging data did not always cover the entire lesion; therefore, obtaining values for the entire region was not always possible. The spatial extent of regions with abnormal MR imaging parameters was, therefore, expressed as a percentage of the portion of the entire T2 lesion that was covered by the PRESS excitation volume.

There was considerable heterogeneity in the distribution of the various abnormal perfusion regions within both grade III and grade IV gliomas as displayed in Fig 1. Volumes of abnormal perfusion regions compared to the T2h region were $16 \%$, $12 \%$, and $5 \%$ for the grade IV lesions and $11 \%, 11 \%$, and $2 \%$ for the grade III lesions (Table 1). In both patient populations, a decreasing trend was observed in normalized mean volume from $\mathrm{aPH}$ to aRec to $\mathrm{aPH}+\mathrm{aRec}$ regions. When compared to the $\mathrm{aPH}+\mathrm{aRec}$ region, the volumes of $\mathrm{aPH}$ and aRec were 


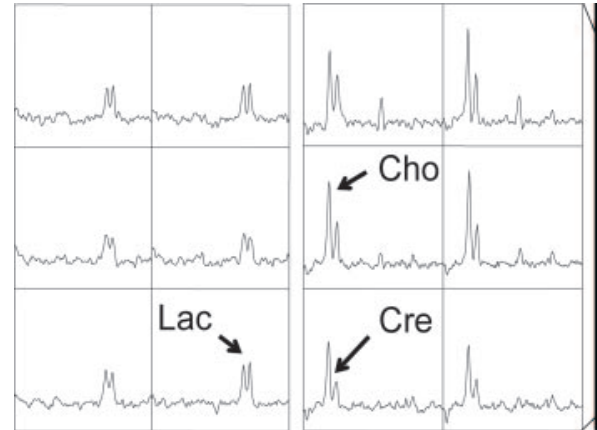

Difference Spectra Summed Spectra

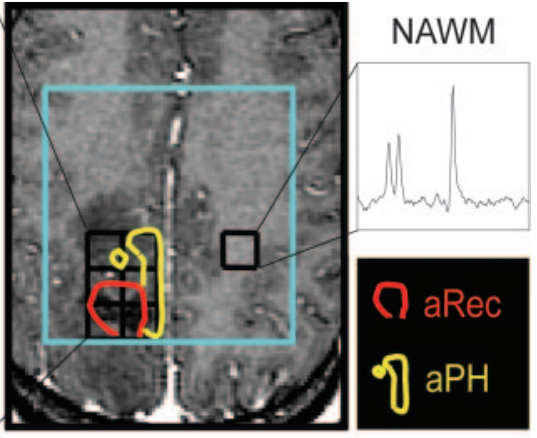

Grade III glioma
Fig 2. Spectral patterns and regions of abnormal perfusion for a representative patient with nonenhancing grade II glioma. From the summed spectra, a clear elevation of choline (Cho), especially in the aPH region (yellow), and a reduction in creatine (Cre) levels, most notably in the aRec region (red), are observed compared with the contralatera NAWM spectra. Increased lactate (Lac) levels that persis throughout the T1-hypointense lesion are shown by the difference spectra.

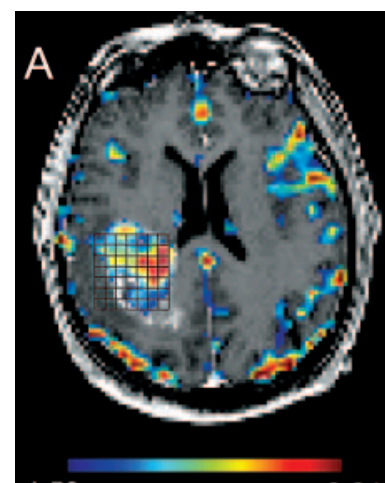

1.59 3.64

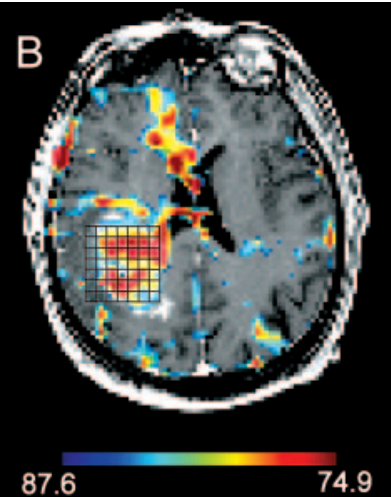

C

74.9

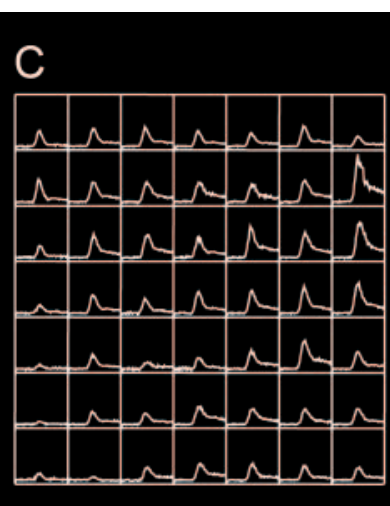

Fig 3. Perfusion-derived nonparametric maps of peak height $(A)$ and percent recovery $(B)$ overlaid on postcontrast T1-weighted images with corresponding $\triangle R 2^{*}$ curves $(C)$ for a representative grade IV glioma. $D-F$, Corresponding metabolite maps of choline $(D)$, creatine $(E)$, and lactate $(F)$.
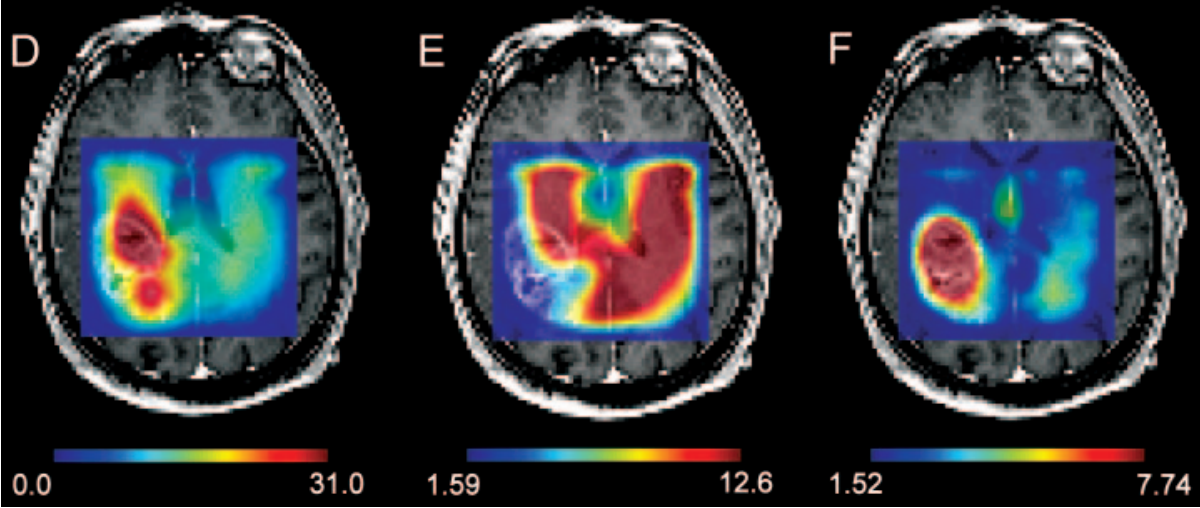

significantly larger for both grade III and grade IV gliomas. However, no statistically significant difference was found between $\mathrm{aPH}$ and aRec. The normalized mean volumes of $\mathrm{aPH}+\mathrm{aRec}$ were significantly larger in patients with grade IV gliomas than in patients with grade III gliomas, with respective values of $5 \%$ and $2 \%$. An increasing trend was observed for aPH volume and aRec volume with grade, but the differences ( $11 \%$ versus $16 \%$ for aPH and $10 \%$ versus $12 \%$ for aRec) were not significant ( $\mathrm{aPH}, P=.2$ and aRec, $P=.1$ ).

Figure 2 shows the spectral patterns and regions of abnormal perfusion for a representative patient with nonenhancing grade III glioma. From the summed spectra, we notice a clear elevation of choline, especially in the aPH region, and a reduction in creatine levels, most notably in the aRec region, compared to the contralateral NAWM spectra. A uniform presence of increased lactate levels throughout the T1-hypointense lesion is observed from the difference spectra. Figure 3 illustrates metabolite maps created from the sum and difference spectra for choline, creatine, and lactate.
Table 2: Mean metabolite levels within abnormal perfusion and T2h regions compared with contralateral NAWM for grade IV gliomas

\begin{tabular}{lcccc}
\hline Region & Choline & Creatine & Lactate & No. Voxels \\
\hline aPH & $17.6 \pm 10.2$ & $9.1 \pm 5.6$ & $4.3 \pm 2.3$ & $54 \pm 43$ \\
aRec & $15.9 \pm 7.7$ & $8.2 \pm 4.7$ & $4.3 \pm 2.7$ & $50 \pm 70$ \\
aPH+aRec & $16.1 \pm 8.6$ & $8.1 \pm 4.9$ & $4.8 \pm 3.3$ & $24 \pm 38$ \\
T2h & $14.4 \pm 6.4$ & $9.0 \pm 4.7$ & $3.4 \pm 2.5$ & $172 \pm 117$ \\
NAWM & $16.4 \pm 4.5$ & $14.5 \pm 3.6$ & $1.2 \pm 1.9$ & N/A \\
\hline
\end{tabular}

Note:-aPH indicates the region of abnormal peak height with normal recovery; aRec, the region of abnormal recovery with normal peak height; NAWM, normal-appearing white matter; N/A, not applicable.

When comparing metabolite levels within the various abnormal perfusion regions with the $\mathrm{T} 2 \mathrm{~h}$ region, we observed different trends for each glioma grade. For grade IV gliomas (Table 2$)$, significantly elevated choline $(P<.02)$ was observed in the aPH region compared to the surrounding pure T2 region, whereas levels of creatine and lactate were similar between these regions $(P>.6$ and $P>.1$, respectively). When 
Table 3: Mean metabolite levels within abnormal perfusion and T2h regions compared with contralateral NAWM for grade III gliomas

\begin{tabular}{lcccc}
\hline Region & Choline & Creatine & Lactate & No. voxels \\
\hline aPH & $18.9 \pm 7.1$ & $12.6 \pm 4.5$ & $2.9 \pm 1.2$ & $31 \pm 24$ \\
aRec & $17.9 \pm 8.0$ & $10.6 \pm 4.2$ & $2.6 \pm 1.5$ & $21 \pm 26$ \\
aPH+aRec & $20.4 \pm 9.5$ & $12.7 \pm 6.0$ & $2.5 \pm 1.9$ & $5 \pm 3$ \\
T2h & $18.9 \pm 6.9$ & $11.5 \pm 3.6$ & $2.9 \pm 1.1$ & $190 \pm 122$ \\
NAWM & $15.2 \pm 4.8$ & $14.8 \pm 4.4$ & $1.4 \pm 1.8$ & N/A \\
\hline
\end{tabular}

Note:-aPH indicates the region of abnormal peak height with normal recovery; aRec, the region of abnormal recovery with normal peak height; NAWM, normal-appearing white matter; N/A, not applicable.

$\begin{aligned} & \text { Table 4: Comparison of mean metabolite levels within abnormal } \\
& \text { perfusion and T2h regions from voxels across all patients of each } \\
& \text { grade }\end{aligned}$
\begin{tabular}{lcccccc}
\multicolumn{2}{c}{ Choline } & & \multicolumn{2}{c}{ Creatine } \\
\cline { 2 - 3 } \cline { 5 - 6 } Region & Grade III & Grade IV & & Grade III & Grade IV \\
\hline aPH & $16.9 \pm 9.6$ & $16.5 \pm 12.1^{*}$ & & $10.7 \pm 5.8$ & $7.6 \pm 5.9^{* *}$ \\
aRec & $17.9 \pm 11.2$ & $16.0 \pm 9.4$ & & $8.8 \pm 5.0$ & $8.2 \pm 4.8$ \\
aPH+aRec & $20.6 \pm 12.8$ & $19.0 \pm 11.7$ & & $11.2 \pm 6.9$ & $8.4 \pm 5.4^{*}$ \\
T2h & $18.9 \pm 10.7$ & $13.6 \pm 8.7$ & & $11.0 \pm 5.6$ & $8.5 \pm 5.5$ \\
\hline
\end{tabular}

Note:- aPh indicates the region of abnormal peak height with normal recovery; aRec, the region of abnormal recovery with normal peak height.

${ }^{*} P<.02$.

${ }^{* *} P<.001$

comparing the aRec to the $\mathrm{T} 2 \mathrm{~h}$ region in these same patients, a significant reduction in creatine and increase of lactate levels were also observed $(P<.05)$, in addition to elevated choline levels $(P<.02)$. Metabolite peak heights for choline, creatine, and lactate within the $\mathrm{aPH}+\mathrm{aRec}$ region were similar to levels observed in the aRec region. All metabolite values within abnormal perfusion and $\mathrm{T} 2 \mathrm{~h}$ regions were significantly different from those in NAWM, with higher creatine and reduced lactate in NAWM. Choline levels in the different abnormal perfusion regions varied compared to those in the NAWM region, however, with elevated choline levels greater than NAWM only present in the aPH region.

For patients with grade III gliomas, no significant differences in metabolite levels were found among regions (Table 3 ), but there was a decreasing trend for creatine in the aRec region compared to all other abnormal perfusion and $\mathrm{T} 2 \mathrm{~h}$ regions. Creatine was elevated in both the $\mathrm{aPH}$ and $\mathrm{aPH}+\mathrm{aRec}$ regions relative to the $\mathrm{T} 2 \mathrm{~h}$ region. Choline and lactate levels were similar in all abnormal perfusion and $\mathrm{T} 2 \mathrm{~h}$ regions, with the exception of a tendency toward increased choline levels in the $\mathrm{aPH}+\mathrm{aRec}$ region. The lack of significance for these trends was most likely due to the smaller number of patients exhibiting abnormal regions in the grade III cohort. All abnormal perfusion and $\mathrm{T} 2 \mathrm{~h}$ regions exhibited significantly increased choline and lactate levels and decreased creatine levels compared to those in the NAWM region.

Lactate levels were significantly elevated in abnormal perfusion regions of grade IV gliomas compared to those in the corresponding grade III region $(P<.02$, Tables 2 and 3$)$. Significant differences in choline and creatine between grades were only observed when combining voxels across all patients (Table 4). With this analysis, creatine levels were significantly reduced for patients with grade IV gliomas in all abnormal perfusion and surrounding T2 hyperintense regions except for the aRec region. Choline levels within the aPH region were significantly elevated in grade III gliomas compared to their grade IV counterparts.

\section{Discussion}

Combining the functional in vivo techniques of MRSI and DSC-MR imaging has allowed us to relate metabolic activity with vascular properties and provide insight into the malignant potential of high-grade gliomas. Through quantitative assessment of perfusion parameters that do not rely on any prior assumptions as to the biology of the model that they describe, we were able to separate first-pass bolus characteristics from the recirculation phase and derive independent regions of increased vessel volume and BBB breakdown. In addition, the 2 spectra-acquisition cycles facilitated the detection of metabolites that have been associated with active tumor proliferation and abnormal cellular energetics that, together with vascular structure, are expected to directly or indirectly influence the level of oxygenation in these tumors. Previous data have shown spatial heterogeneity when comparing percent recovery and peak-height values obtained from a nonparametric model for both the region of contrast enhancement and the surrounding region of hyperintensity on T2weighted images. ${ }^{30}$ Our goal was to gain further insight into the metabolic changes occurring within both areas of wellperfused tumor and regions where the BBB was compromised, in addition to identifying regions that could potentially differentiate between the grade III and grade IV lesions. Whereas the presence of such heterogeneity is not surprising based on the histologic characteristics of these tumors, identifying coincident regions of leaky or dense vasculature and markers of poor oxygenation and cellular proliferation could be useful in determining the more aggressive part of the tumor for planning and monitoring therapy.

When the vascular supply is no longer adequate to support the increasing metabolic demands of the rapidly proliferating tumor cells, there is an increased reliance on glycolysis for energy production, and this promotes the formation of new blood vessels. Insufficient perfusion of oxygen from the capillary bed to the surrounding tissue is also caused by tortuous, degraded, and abnormal tumor vasculature. We limited our regions to only abnormal peak height with normal recovery $(\mathrm{aPH})$, only abnormal recovery with normal peak height (aRec), and both abnormal peak height and recovery $(\mathrm{aPH}+$ aRec) to relate the respective spatial distribution of elevated vessel volume and abnormal recirculation/leakage with metabolic activity, without the confounding effect of tortuosity, which is likely to be manifested as an increase in both parameters and to be in the $\mathrm{aPH}+\mathrm{aRec}$ region. A $5 \times 5 \mathrm{~mm}$ in-plane resolution was selected as an intermediate value to ensure adequate signal to noise of the perfusion data, avoid overinterpolation of metabolite maps, facilitate image registration, and not compromise spatial resolution in the definition of our region boundaries, which will eventually be incorporated into treatment planning. The abnormal range of percent recovery and peak-height values was determined on the basis of previous analysis of the relativity curves from normal tissue. ${ }^{30}$

Grade IV gliomas exhibited the highest choline levels, significantly greater than values found in NAWM in the $\mathrm{aPH}$ region and suggested increased cell density and/or proliferation. Because increased oxygenation from excess vessels would 
be available to support the additional and/or rapidly dividing cells, an increase in choline with stable creatine and lactate levels is expected for this region. Within the aRec region, the elevation in choline was accompanied by reductions in creatine and increases in lactate. These changes may reflect the dependence of oxygenation on vascular integrity, whereas leaky vessels prevent adequate perfusion of oxygen to the surrounding tissue, resulting in anaerobic respiration, the formation of lactate, and lower energy reserves. All metabolite levels within the $\mathrm{aPH}+\mathrm{aRec}$ region were similar to those observed in the aRec region, suggesting the presence of leaky vasculature rather than vessel tortuosity.

In grade III gliomas, creatine was the only metabolite that showed differences between abnormal perfusion regions. Decreasing trends were observed in the aRec region, whereas both the $\mathrm{aPH}$ and $\mathrm{aPH}+\mathrm{aRec}$ regions exhibited increasing trends. This may mean that more energy is needed in the initial recruitment and formation of new vessels. None of these trends were significant, however, due to the smaller region sizes and smaller population that was available in the study. The reduction in creatine levels in the aRec region compared to the surrounding pure T2 hyperintensity experienced by patients with grade III gliomas is consistent with previous findings of lower creatine values for patients with grade III gliomas in regions of contrast enhancement ${ }^{37}$ and supports creatine as a marker for hypoxia, though this reduction may be due to an overall metabolite depression from evolving necrotic tissue.

Grade IV gliomas exhibited significantly elevated lactate levels compared to grade III gliomas in all abnormal perfusion regions. In patients with grade III gliomas, the presence of lactate did not appear to be localized to any particular region, and the amount of lactate in all abnormal perfusion regions was consistent with levels found in the surrounding T2 hyperintensity. This finding agreed with previously published data showing no significant correlation between volumes of elevated lactate and any morphologic abnormality. ${ }^{20}$

Our results suggest that a reduction in creatine levels and the presence of regions with abnormal vasculature may be considered as noninvasive markers of tumor progression from grade III to grade IV gliomas. Increased cellular proliferation, as indicated by elevated choline in the aPH region of grade III gliomas, may initially cause dilation of existing blood vessels and/or the formation of new ones. This mitosis-induced angiogenic phenomenon is supported by previous studies that demonstrated a positive correlation between vessel volume and choline levels. ${ }^{38}$ The new vessels that are formed often lack the complex structure of the normal brain vasculature, resulting in increased endothelial permeability. Although diminished creatine levels were observed in regions of microvascular leakage for both glioma grades, lactate levels were only elevated in grade IV tumors. This finding implicates creatine as an early biomarker of reduced energy reserves from anaerobic respiration before lactate accumulation. We hypothesize that once transformation to a more malignant phenotype occurs, the decline of creatine becomes widespread and lactate levels begin to rise, especially in regions with leaky vessels.

An alternative explanation is that the existence of lactate is a marker of hypoxia, which induces angiogenesis in tu- mors. ${ }^{1,9,31}$ The presence of lactate is the result of heightened glycolytic metabolism within hypoxic regions that are driven by increased proliferation. Li et al found higher rCBV values within regions of elevated lactate and a positive correlation between maximal peak height and volume of lactate of highgrade tumors. ${ }^{21,31}$ In that case, vessel wall permeability was not assessed, and there was no distinction between the 2 tumor grades. The fact that we only observed elevated lactate and reduced creatine within the regions of abnormal recovery suggests that $\mathrm{BBB}$ breakdown, originating from damage to the existing vasculature, plays a role in oxygen depletion. It seems likely that once the angiogenic switch is activated, the oxygenation process is restored as reflected by our results of similar creatine and lactate levels in the aPH region and the surrounding nonenhancing T2 lesion. The fact that all metabolite levels within the $\mathrm{aPH}+\mathrm{aRec}$ region were similar to those observed in the aRec region further substantiates the importance of vessel integrity in oxygenation. It is possible that the correlation between lactate and rCBV observed previously may be due to elevated rCBV measurements and microvascular leakage.

Although the clinical role of $3 \mathrm{D}^{1} \mathrm{H}-\mathrm{MRSI}$ and DSC-MR imaging requires further evaluation, the current study has shown that these 2 techniques are useful in adding specificity to characterizing spatial heterogeneity in high-grade gliomas. However, limitations of this study include the small sample size (especially for the grade III population), the heterogeneous nature of these tumors, and the lack of spatial correlation between imaging parameters and histopathology from the surgically resected material. Other possible confounding factors include the administration of steroids to relieve intracranial pressure and mass effect, though these effects should only manifest as decreased volumes of region abnormality.

Assessment of the level of oxygenation in tumors is becoming increasingly important because hypoxia has been shown to be a predictor of aggressive disease, metastatic spread of tumor cells, and poor response to radiation therapy and chemotherapy. ${ }^{8,39}$ Parametric maps of elevated peak height, choline, and lactate levels, and abnormal recovery and creatine levels, may be beneficial in treatment planning, including target definition for conformal beam radiation therapy and biopsy guidance to help avoid underestimation of the histologic grading of nonenhancing tumors. ${ }^{39-41}$ Predicting hypoxic regions would be especially advantageous in determining the most radiosensitive region of the tumor that would respond best to treatment. Future assessment of the role of the combination of these vascular and metabolic parameters as possible indicators of the effectiveness of novel antiangiogenic therapies, as well as their ability to predict survival and regions of recurrence, would be of great interest.

\section{Conclusions}

The spatial distributions of tumor microvasculature and metabolic characteristics have shown considerable heterogeneity in patients with grade III and grade IV gliomas. Key changes in regions of abnormal perfusion are increased choline, decreased creatine, and increased lactate. Depressed creatine and elevated lactate levels confirmed the lack of oxygenation within regions of compromised vascular integrity. Future studies will assess the prognostic value of these perfusion and 
metabolic parameters in both predicting and evaluating tumor response to antiangiogenic therapy for patients with high-grade glioma.

\section{Acknowledgments}

We thank Michael C. Lee, Esin Ozturk-Isik, Rebeca Choy, and Jamie Chang of the Department of Radiology, University of California, San Francisco, for their assistance with data analysis or helpful comments regarding this manuscript.

\section{References}

1. Allen N. Oxidative metabolism of brain tumors. Prog Exp Tumor Res 1972;17:192-209

2. Amoroso A, Del Porto F, Di Monaco C, et al. Vascular endothelial growth factor: a key mediator of neoangiogenesis-a review. Eur Rev Med Pharmacol Sci 1997;1:17-25

3. Damert A, Machein M, Breier G, et al. Up-regulation of vascular endothelial growth factor expression in a rat glioma is conferred by two distinct hypoxiadriven mechanisms. Cancer Res 1997;57:3860-64

4. Wesseling P, Ruiter DJ, Burger PC. Angiogenesis in brain tumors: pathobiological and clinical aspects. J Neurooncol 1997;32:253-65

5. Schneider SW, Ludwig T, Tatenhorst L, et al. Glioblastoma cells release factors that disrupt blood-brain-barrier features. Acta Neuropathol (Berl) 2004;107:272-76

6. Jain RK. Vascular and interstitial barriers to delivery of therapeutic agents in tumors. Cancer Metastasis Rev 1990;9:253-66

7. Alger JR, Frank JA, Bizzi A, et al. Metabolism of human gliomas: assessment with H-1 MR spectroscopy and F-18 fluorodeoxyglucose PET. Radiology 1990;177:633-41

8. Evans SM, Koch CJ. Prognostic significance of tumor oxygenation in humans. Cancer Lett 2003;195:1-16

9. Herholz K, Heindel W, Luyten PR, et al. In vivo imaging of glucose consumption and lactate concentration in human gliomas. Ann Neurol 1992;31:319-27

10. Gillies RJ, Morse DL. In vivo magnetic resonance spectroscopy in cancer. Annu Rev Biomed Eng 2005;7:287-326

11. Howe FA, Barton SJ, Cudlip SA, et al. Metabolic profiles of human brain tumors using quantitative in vivo $1 \mathrm{H}$ magnetic resonance spectroscopy. Magn Reson Med 2003;49:223-32

12. McBride DQ, Miller BL, Nikas DL, et al. Analysis of brain tumors using $\mathbf{1 H}$ magnetic resonance spectroscopy. Surg Neurol 1995;44:137-44

13. Nelson SJ, Vigneron DB, Dillon WP. Serial evaluation of patients with brain tumors using volume MRI and 3D 1H MRSI. NMR Biomed 1999;12:123-38

14. Nelson SJ. Analysis of volume MRI and MR spectroscopic imaging data for the evaluation of patients with brain tumors. Magn Reson Med 2001;46:228-39

15. Gill SS, Thomas DG, Van Bruggen N, et al. Proton MR spectroscopy of intracranial tumours: in vivo and in vitro studies. J Comput Assist Tomogr 1990;14:497-504

16. Li X, Lu Y, Pirzkall A, et al. Analysis of the spatial characteristics of metabolic abnormalities in newly diagnosed glioma patients. J Magn Reson Imaging 2002;16:229-37

17. Jackson A, Jayson GC, Li KL, et al. Reproducibility of quantitative dynamic contrast-enhanced MRI in newly presenting glioma. $\mathrm{Br} J$ Radiol 2003;76:153-62

18. Law M, Cha S, Knopp EA, et al. High-grade gliomas and solitary metastases: differentiation by using perfusion and proton spectroscopic MR imaging. $R a$ diology 2002;222:715-21

19. Law M, Yang S, Wang H, et al. Glioma grading: sensitivity, specificity, and predictive values of perfusion MR imaging and proton MR spectroscopic imaging compared with conventional MR imaging. AJNR Am J Neuroradiol 2003;24:1989-98

20. Catalaa I, Henry R, Dillon WP, et al. Perfusion, diffusion and spectroscopy values in newly diagnosed cerebral gliomas. NMR Biomed 2006;19:463-75
21. Li X, Vigneron DB, Lupo JM, et al. Relationship between lactate, choline, creatine, and perfusion parameters in newly diagnosed high grade gliomas. Proceedings from the 12th Annual International Society for Magnetic Resonance in Medicine Meeting. May 12-17, 2004; Kyoto, Japan

22. Fayed N, Modrego PJ. The contribution of magnetic resonance spectroscopy and echoplanar perfusion-weighted MRI in the initial assessment of brain tumours. J Neurooncol 2005;72:261-65

23. Cha S, Knopp EA, Johnson G, et al. Intracranial mass lesions: dynamic contrast-enhanced susceptibility-weighted echo-planar perfusion MR imaging. Radiology 2002;223:11-29

24. Aronen HJ, Gazit IE, Louis DN, et al. Cerebral blood volume maps of gliomas: comparison with tumor grade and histologic findings. Radiology 1994;191:41-51

25. Griebel J, Mayr NA, de Vries A, et al. Assessment of tumor microcirculation: a new role of dynamic contrast MR imaging. J Magn Reson Imaging 1997;7:111-19

26. Guckel F, Brix G, Rempp K, et al. Assessment of cerebral blood volume with dynamic susceptibility contrast-enhanced gradient-echo imaging. J Comput Assist Tomogr 1994;18:344-51

27. Knopp EA, Cha S, Johnson G, et al. Glial neoplasms: dynamic contrast-enhanced T2*-weighted MR imaging. Radiology 1999;211:791-98

28. Provenzale JM, Wang GR, Brenner T, et al. Comparison of permeability in high-grade and low-grade brain tumors using dynamic susceptibility contrast MR imaging. AJR Am J Roentgenol 2002;178:711-16

29. Law M, Yang S, Babb JS, et al. Comparison of cerebral blood volume and vascular permeability from dynamic susceptibility contrast-enhanced perfusion MR imaging with glioma grade. AJNR Am J Neuroradiol 2004;25:746-55

30. Lupo J, Cha S, Chang SM, et al. Dynamic susceptibility-weighted perfusion imaging of high-grade gliomas: characterization of spatial heterogeneity. AJNR Am J Neuroradiol 2005;26:1446-54

31. Li X, Vigneron DB, Cha S, et al. Relationship of MR-derived lactate, mobile lipids, and relative blood volume for gliomas in vivo. AJNR Am J Neuroradiol 2005;26:760-69

32. Star-Lack J, Spielman D, Adalsteinsson E, et al. In vivo lactate editing with simultaneous detection of choline, creatine, NAA, and lipid singlets at $1.5 \mathrm{~T}$ using PRESS excitation with applications to the study of brain and head and neck tumors. J Magn Reson 1998;133:243-54

33. Li X, Graves EE, Vigneron DB, et al. Reliable estimate of lactate and lipid for newly-diagnosed glioma patients using lactate-edited 3D 1H-MRSI with ellipsoidal k-space sampling. Proceedings from the 11th Annual International Society for Magnetic Resonance in Medicine Meeting. July 2003; Toronto, ON, Canada

34. Tran TK, Vigneron DB, Sailasuta N, et al. Very selective suppression pulses for clinical MRSI studies of brain and prostate cancer. Magn Reson Med 2000;43:23-33

35. Rueckert D, Sonoda LI, Hayes C, et al. Nonrigid registration using free-form deformations: application to breast MR images. IEEE Trans Med Imaging 1999;18:712-21

36. Studholme C, Hill D, Hawkes D. An overlap invariant entropy measure of 3D medical image alignment. Pattern Recognit 1999;32:71-86

37. Ozturk E, Chang SM, Nelson SJ. 3D 1H MRSI, MRI, and diffusion tensor imaging in newly-diagnosed patients with grade 3 brain tumors. Proceedings from the 12th Annual International Society for Magnetic Resonance in Medicine Meeting. May 15-21, 2004; Kyoto, Japan

38. Henry RG, Vigneron DB, Fischbein NJ, et al. Comparison of relative cerebral blood volume and proton spectroscopy in patients with treated gliomas. AJNR Am J Neuroradiol 2000;21:357-66

39. Pirzkall A, McKnight TR, Graves EE, et al. MR-spectroscopy guided target delineation for high-grade gliomas. Int $J$ Radiat Oncol Biol Phys 2001;50:915-28

40. Nelson SJ, Graves E, Pirzkall A, et al. In vivo molecular imaging for planning radiation therapy of gliomas: an application of $1 \mathrm{H}$ MRSI. J Magn Reson Imaging 2002;16:464-76

41. Nelson SJ. Magnetic resonance spectroscopic imaging: evaluating responses to therapy for gliomas. IEEE Eng Med Biol Mag 2004;23:30 -39 